\title{
Pilot study: Computer-based virtual anatomical interactivity for rehabilitation of individuals with chronic acquired brain injury
}

\author{
C. Douglas Simmons, PhD, OTR/L, FAOTA; ${ }^{*}$ Sajay Arthanat, PhD, OTR/L, ATP; ${ }^{2}$ Vincent J. Macri, BA, MA \\ ${ }^{1}$ Salem State University, Salem, MA; ${ }^{2}$ University of New Hampshire, Durham, $\mathrm{NH} ;{ }^{3}$ Independent Practitioner
}

\begin{abstract}
Deficiencies in upper-limb motor function and executive functioning can compromise an affected individual's ability to complete everyday activities. Impaired motor and executive functioning therefore pose a risk to increasing numbers of veterans who have been diagnosed with acquired brain injury. This article reports on changes in upper-limb motor function and executive functioning of 12 adult participants with chronic acquired brain injury using a novel, computer-based, motor and cognitive rehabilitation program called PreMotor Exercise Games (PEGs). Manual muscle, goniometric range of motion, and dynamometer assessments were used to determine motor functioning while the Executive Function Performance Test measured cognitive functioning. A three-level repeated measures design was conducted to determine changes pre- and postintervention. Participants demonstrated significant improvement in shoulder $(p=0.01)$ and wrist $(p=0.01)$ range of motion and clinically relevant improvement for elbow range of motion. Participants demonstrated clinically relevant improvement in shoulder, elbow, and wrist strength. Finally, participants demonstrated significant improvement in executive functioning $(p<$ 0.05 ). Using PEGs as a modality for both motor and cognitive intervention is a potentially beneficial adjunct to rehabilitation and warrants further study.
\end{abstract}

Key words: acquired brain injury, community health, executive functioning, motor control, motor imagery, motor relearning, neurological rehabilitation, PreMotor Exercise Games, stroke, virtual rehabilitation.

\section{INTRODUCTION}

The demographic shift caused by an aging population in the United States poses new challenges to the healthcare industry, including the treatment of acquired brain injury (ABI) due to stroke and traumatic brain injury (TBI), which are often related to aging, cardiovascular disease, and falls. $\mathrm{ABI}$ refers to a type of brain injury that can result from an impact to the brain, including TBI and stroke [1]. The Centers for Disease Control and Prevention's National Center for Injury Prevention and Control estimates that 5.3 million U.S. citizens, or 2 percent of the population, are living with disability from ABI [2]. Strokes are the leading cause of long-term disability in the United States, and it is estimated that 795,000 strokes occur annually. The annual incidence of TBI in the United States is approximately 1.7 million [3]. Over 80 percent of individuals with $\mathrm{ABI}$ have motor problems, which affect the functional use of the upper limb [4].

\footnotetext{
Abbreviations: $\mathrm{ABI}=$ acquired brain injury, $\mathrm{ANOVA}=$ analysis of variance, EFPT $=$ Executive Function Performance Test, $\mathrm{fMRI}=$ functional magnetic resonance imaging, MVPT = Motor-Free Visual Perception Test, PEG $=$ PreMotor Exercise Game, $\mathrm{RCT}=$ randomized control trial, $\mathrm{ROM}=$ range of motion, $\mathrm{TBI}=$ traumatic brain injury.

*Address all correspondence to C. Douglas Simmons, PhD, OTR/L, FAOTA; Salem State University, 352 Lafayette St, Salem, MA 01970-5353; 978-542-2389.

Email: csimmons@salemstate.edu

http://dx.doi.org/10.1682/JRRD.2013.05.0103
} 
Specifically, 6 mo after stroke, only 38 percent of patients recover limited dexterity in the paretic arm and only 12 percent recover substantial function despite having received rehabilitation [5]. Therefore, it is not surprising that novel rehabilitative modalities are emerging to address upperlimb dysfunction for adults with ABI. Some of these potential approaches to promote recovery of movement of the upper limb are based on the discovery of mirror neurons. For example, interventions such as motor imagery, mirror therapy, action-observation therapy, or computer-based therapy for the upper limb may derive in part from the mirror neuron theory described here [6-10].

\section{Mirror Neurons}

Mirror neurons were originally thought to be located in the lateral premotor cortex of the brain but are now known to be more widespread and most likely exist in the posterior parietal cortex and the premotor cortex. The mirror neuron hypothesis postulates that these neurons are active not only when individuals execute motor actions but also when they observe, imagine, or listen to these same actions [11]. In addition, the relearning of impaired cortical motor function may be mediated by the mirror neuron system. Mirror neurons may underlie the basis for how humans learn to connect motor behavior with cognitive and emotional intention [11-12]. For example, Gutman et al. stated, "mirror neurons may allow us to see a motor behavior and link it to the cognitive and emotional intention of the performer" [13].

Mirror neuron research has encouraged the development of new motor rehabilitation approaches. In addition, advances in neuroimaging have revealed that rehabilitation induces brain plasticity after stroke for patients during the chronic stages of recovery [14]. New rehabilitation paradigms have therefore incorporated the use of motor imagery (physically or computer generated) to facilitate mirror neuron activation. Such therapies derive from simulation theory, which states that within the central nervous system there is functional correspondence between movement imagery, movement observation, and actual movement $[6,15]$. Research, which has supported these theories, has led to application in rehabilitative strategies such as mirror therapy, computer-based motor learning/motor imagery, and action-based therapy.

\section{Mirror Therapy}

Mirror therapy evolved from the discovery, theoretical development, and research associated with mirror neurons and motor recovery, specifically after limb amputation and accompanying brain trauma. It is based on visual and physical simulation and stimulation. During mirror therapy, a mirror is placed in a patient's mid-sagittal plane reflecting the noninvolved side as if it were the involved side. By using this setup, movements of the noninvolved limb create the visual simulation, or false appearance, of normal movements of the involved side [16]. Michielsen et al., through functional magnetic resonance imaging (fMRI), found that during this process the precuneus and posterior cingulate cortex were activated [17]. Research demonstrates the need for continual growth for this type of therapy based on the observed benefits. Improvements such as enhanced fluidity of movement patterns, ability to carry out daily activities, pain reduction, improved grasp, and maintenance were demonstrated to extend beyond 6 mo of treatment [17-20]. Another recent study using mirror therapy, published by Lee et al. in 2012, noted improvements in Fugl-Meyer Assessment scores for shoulder, elbow, and hand, as well as improvement in the Manual Function Test scores for shoulder and hand items [21].

\section{Computer-Based Intervention}

With the integration of digital technology into rehabilitative medicine, researchers and therapists began to incorporate mirror therapy concepts into computer programing for upper-limb treatment after ABI [22]. These new therapeutic modalities have resulted in decreased demand on therapists' time, with a reduction of mass practice approaches and an increase in the time patients can spend working on upper-limb motor functioning [23]. The concepts of mirror therapy have been integrated into computer-based programming in which the patient may use a noninvolved limb to manipulate objects, including virtual involved limbs, on the computer screen. These therapies enable patients to visualize movement with the assistance of a virtual, computer-simulated involved limb, which allows them to simultaneously create and observe that movement on screen. Wille et al. found that patients' motivation for this type of therapeutic programming was high and study participants demonstrated improved hand function such as grasp and release [23].

Because of the demands of computer interfaces requiring input methods, such as a joystick or mouse, researchers also observed that mental practice and mental imagery training were occurring. Therefore, the transition of mirror therapy from a table-top approach to a computer-based 
approach brought multiple environmental stimulants, such as mental practice, motor imagery, and mirror therapy concepts, into the therapeutic process of motor recovery for the upper limb following ABI [24]. Butler and Page defined mental practice with motor imagery as an "internal simulation of movements involving one's own body in the absence of overt execution" [24]. Studies using fMRI techniques have reported activation of the supplementary motor area, premotor cortex, primary motor cortex, parietofrontal circuitry, temporal gyrus, and ipsilateral anterior lobe of the cerebellum [24-26].

Research associated with mirror therapy, mental imagery, mental practice, and action-observation therapy has experienced accelerated development in recent years. The vast majority of research involving rehabilitation has been centered on adult patients with stroke in the acute stages of recovery. Calayan and Dizon completed a systematic review on the effectiveness of mental imagery and mental practice and reported that those studies using a randomized control trial (RCT) design found improvements in upper-limb movement for both range of motion (ROM) and strength, as well as reaching and grasp during functional tasks for individuals $4 \mathrm{wk}$ poststroke [27]. In a similar review, Braun et al. reviewed 10 studies that included several RCT and single case-study designs: 8 studies found significant outcomes for these therapeutic approach on patients with stroke [28]. Studies that included fMRI found that during mental imagery and mental practice, plasticity is upregulated in the hemisphere opposite to the lesion for participants with stroke $[25,29]$.

Although a majority of research studies have involved patients in the acute stages of stroke, several studies postulated that computer-based interventions promote neuroplasticity and could be effective even years after central nervous system damage and suggest a need for continued research [24,28,30-31]. Research associated with computer-based intervention incorporating mirror therapy, mental imagery, and mental practice has been conducted primarily in the pediatric setting and is limited. In addition, no studies were found that considered the assumptions of Gutman et al. [13] that a strong cognitive demand is present as participants consider the intent and purpose of movement. Therefore, the pilot study described herein sought to measure both motor recovery and cognitive enhancement of an adult population in the chronic stage of recovery from ABI. Specifically, this study proposed to answer the following research questions:
1. Will participation in a computer-based intervention for the upper limb improve ROM on the involved side for shoulder, elbow, and wrist flexion in adults with chronic ABI?

2. Will participation in a computer-based intervention for the upper limb improve strength on the involved side for shoulder, elbow, and wrist flexion in adults with chronic ABI?

3. Will participation in a computer-based intervention for the upper limb improve executive functioning skills in adults with chronic $\mathrm{ABI}$ ?

\section{METHODS}

\section{Design}

This intervention study was conducted using a threelevel repeated measures design. The first two measures were taken at baseline levels with the intent of verifying stability of outcome variables. The interval between the first and second baselines was 2 wk. PreMotor Exercise Games (PEGs) intervention, using the 3D PreMotorSkill Technology (Durham, New Hampshire), was subsequently provided, and the third series of measures (postintervention) was gathered immediately following intervention. The first and second authors were independent researchers in adult neurological rehabilitation who planned and implemented the study. The third author assisted with the technical implementation of the intervention, provided feedback on PEGs development, contributed to the technological detail of this article, and did not play any role in the design, analysis, and interpretation of results.

The research design was well suited to the nature of this pilot study in terms of time, budget, and availability of study participants and personnel. The relevance and key merits of repeated measures design in this research were (1) minimizing between-subject variability (a concern with individuals recovering from brain injury), (2) accounting for the within-subject variability (changes in individual subjects over time), and (3) precluding the need for a control group since study participants themselves serve as a time-tested control group between the period from baseline to postintervention [32].

\section{Setting}

The study was conducted at a community center day program for individuals with $\mathrm{ABI}$. Institutional review for human subject protection was conducted and approval 
was obtained from the center and through the Research Integrity Services at the University of New Hampshire.

\section{Participants and Recruitment}

Fourteen individuals who experienced various forms of ABI volunteered. All were over $21 \mathrm{yr}$ of age and in chronic stages of neurological recovery. The average chronic duration postinjury was $11.4 \mathrm{yr}$ and average age was $53.9 \mathrm{yr}$. Two volunteers left the study for personal reasons unrelated to participation in the study. The sample size was estimated based on the scope, timeline, and feasibility of the research protocol. Nevertheless, a power analysis based on $f$-test for analysis of variance (ANOVA) indicated that the sample size was reasonably adequate. Anticipating a medium $f$ value of $0.50,14$ participants were required to show statistically significant differences in motor scores (power $=0.80$, $\mathrm{df}_{\mathrm{b}}=2$, and type $\mathrm{I}(\alpha)$ error $\left.=0.05\right)$ for the three data points in our study [33].

All potential participants underwent a screening protocol to confirm their eligibility in the study. Inclusion criteria for the screening were being medically stable (when examined by professional therapists overseeing their daily activities), presence of motor deficits as ascertained by a Quick Functional Range and Strength Assessment, and willingness and availability to participate in the study protocol.

As exclusion criteria, participants in the program were not considered if they presented with potential difficulties in following the intervention protocol as determined by the Motor-Free Visual Perception Test (MVPT) and the Executive Function Performance Test (EFPT). Participants who scored below the 15 th percentile on the MVPT were deemed to have visual-spatial difficulties that would limit their ability to effectively engage in the intervention and therefore were excluded from the study. The EFPT was administered to all potential participants to screen for difficulties in cognition and information processing that would limit their ability to follow the instructions of the intervention and research protocol. In addition, those members with a history of photosensitive epileptic seizures were not considered for inclusion in this study because of safety reasons. In order to ensure that the study results would reflect PEGs play alone, all participants were instructed to and confirmed they would not play any video games, except those designed for therapeutic intervention, throughout the intervention period and postintervention assessments/measurements.

\section{Instruments}

The methods used to record motor skill variables included (1) manual muscle testing with a 0 to 5 rating, with 0 being no strength to 5 being full strength; (2) ROM assessment using goniometer; (3) hand grip strength measurement using a calibrated dynamometer; and (4) pinch strength for key, lateral, and three-jaw chuck (tripod) grasps using a pinch meter.

The EFPT is a highly used assessment of executive functioning for people with $\mathrm{ABI}$ and was used to examine changes in cognition and its consequent effect on daily functioning. The EFPT measures an individual's executive functioning relevant to four independent living skills: (1) simple cooking, (2) telephone use, (3) medication management, and (4) bill payment. The assessment measured executive functioning components consisting of initiation of task, organization, sequencing, safety and judgment, and completion of task. The components are measured across the performance of the four independent living skills. The test produced three levels of scoring: the executive function component score, the independent living skill task score, and a total score. The executive function component score is calculated by summing the numbers recorded on each of the four tasks of the previously listed functional components. Scores on each executive function component can range from 0 to 5 , and therefore, the total for all four tasks can range from 0 to 20 . The independent living skill task score is calculated by summing the five scores of each task. The range for each task is 0 to 25 . The total score is the sum of the performance on all four independent living skill tasks and can range from 0 to 100 . The EFPT uses reverse scoring, with low scores representing better performance on the assessment. The interclass correlation coefficient for interrater reliability is 0.91 , and internal consistency was high ( $\alpha=0.94)$. Construct, concurrent, and criterion validity for the EFPT are strong, as reported by Baum et al. [34].

\section{INTERVENTION}

The 3D PreMotor Skill PEG intervention was conducted by two trained occupational therapy graduate students and directed by a licensed occupational therapist with expertise in neurological rehabilitation. The 3D PreMotor Skill PEG technology is currently in the research and development phase, and as development occurs information will be available via the Internet for clinicians. Instruction in delivery of the 3D PreMotor Skill 
intervention along with assistance was provided to graduate students throughout the intervention process. Initial instruction and guidance were provided, usually taking 5 min or less for participants to become capable of playing PEG simulations. Participants progressed independently thereafter. The design of the PEGs platform accommodates many ABI performable levels of virtual simulation interactivities, meaning that progression of PEG playing is self-directed.

PEGs are based on the theoretical foundation that for individuals with disabled or dysfunctional use of upper limbs, which impede control of purposeful actions or related cognitive processes, imagined action alone results in imagined feedback. Visualization and imagery alone are only somewhat sufficient for rehabilitating action planning, execution, or restoration of unaffected physical actions.

PEGs provide video game-like opportunities so that users are able to instantiate abstract mental representations of physical actions into actual visual displays of simulated physical actions. PEGs include anatomically realistic upper limbs with analogous true ROM that are controllable using the uninvolved limb and any input means, e.g., a standard mouse, touchscreen, or head movements. Upper-limb PEGs exercises included participant-players controlling any or all parts of a virtual affected hand, lower or upper arm (right or left), executing flexion/extension, supination/ pronation, and abduction/adduction in any direction. Participant-players managed displays of some of, the majority of, or all of a virtual upper limb from substantially any angle.
PEG virtual hand games for preaction training consists of (1) pincer action to grasp a key; (2) two finger action to grasp a ball and drop it into a cup; (3) multifinger action to pick up a spoon and drop it into a cup; (4) full hand grasp around a mug handle; (5) tapping actions by index and middle fingers on a remote controller; and (6) hand grasp of objects shaped as stars, circles, or squares, then placement into the corresponding slots (Figure 1(a-b)). These actions were embedded into the following PEGs that simulated real-life tasks: (1) opening a correct box, (2) nine box game with voice instructions to the player, (3) light bulb game, (4) jigsaw puzzle games, (5) simple number game, and (6) simple letter games.

\section{Data Collection}

Data were collected twice in the baseline phase, $2 \mathrm{wk}$ apart, and later following the interventions. All instruments were administered by a licensed occupational therapist who was not involved in promoting intervention.

\section{Data Analysis}

The data were analyzed using descriptive and inferential statistics. Mean scores of outcome measures were plotted on charts for visual analysis. Because of two participant drop-outs and high variability in the data, we chose to closely analyze the response to intervention for each participant and used nonparametric Friedman repeated measures of ANOVA for the sample data to verify statistically significant changes (Type I $\alpha<0.05$ ) from

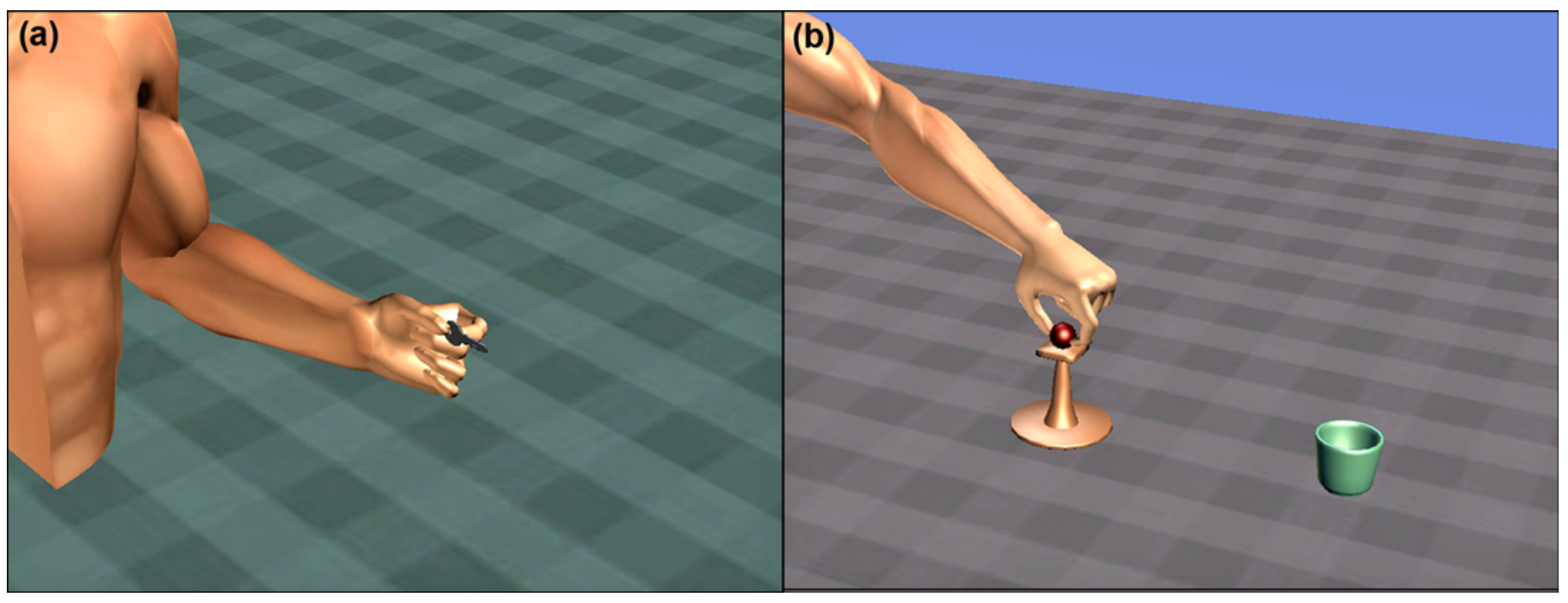

Figure 1.

(a) Grasping and turning key. (b) Two finger action to grasp ball and drop it into cup. 
baseline to postintervention. In addition, we used Pearson correlation to ascertain whether changes in outcome measures were associated with time spent with intervention.

\section{RESULTS}

Table 1 displays the demographic and diagnostic details of the participants. As previously stated, 2 of the 14 recruited participants dropped out of the study because of ongoing health complications and subsequent problems with their availability for the intervention protocol.

The 12 participants were in the age range of 43 to $66 \mathrm{yr}$, with the average age being $53.9 \pm 6.5$ yr. They included eight males and four females. In terms of their nature of brain injury, eight participants had experienced a stroke and four had a TBI, with average time since onset of injury at $11.4 \pm 10.0 \mathrm{yr}$ for all participants.

Table 2 indicates the time spent by each participant on the PEG interventions varied based on participant levels of fatigue, concentration, and transportation challenges in getting to the community center.

\section{Range of Motion}

Figure 2 illustrates the changes in ROM of upper-limb joints for participants from baseline to postintervention for the three upper-limb joints, while Table 3 displays the change in ROM for each participant within the baseline and postintervention phases.

Table 1.

Participant demographics and diagnostic information.

\begin{tabular}{lclcc}
\hline Participant & Age (yr) & Sex & $\begin{array}{c}\text { Nature } \\
\text { of Brain } \\
\text { Injury }\end{array}$ & $\begin{array}{c}\text { Years } \\
\text { Since } \\
\text { Onset }\end{array}$ \\
\hline 1 & 64 & Male & CVA & 8 \\
2 & 54 & Female & TBI & 34 \\
3 & 48 & Male & TBI & 28 \\
4 & 66 & Male & CVA & 9 \\
5 & 50 & Female & TBI & 8 \\
6 & 53 & Male & CVA & 5 \\
7 & 56 & Male & CVA & 7 \\
8 & 55 & Female & TBI & 12 \\
9 & 43 & Male & CVA & 17 \\
10 & 52 & Male & CVA & 2 \\
11 & 49 & Female & CVA & 4 \\
12 & 57 & Male & CVA & 3 \\
\hline CVA = cerebrovascular accident, & TBI = traumatic brain injury. \\
\hline
\end{tabular}

\section{Shoulder Flexion Range of Motion}

The ROM for the 12 participants remained relatively stable from baseline 1 to baseline 2, while at postintervention notable gains in ROM were recorded for participants $3\left(65^{\circ}\right), 8\left(68^{\circ}\right)$, and $11\left(53^{\circ}\right)$. An increasing trend in ROM was noted in participants 7 and 12 across baseline to postintervention. Shoulder ROM was stable for participants 1 and 2, while a slight decline in ROM was observed in participant 4. Shoulder ROM for participants 5,6 , and 9 could not be assessed throughout the study. The study protocol specified that only active range would be measured; if a participant could not actively move a joint they were assigned a score of $0^{\circ}$. Participants 5,6 , and 9 had no active movement for any assessment finding and therefore no score was given. For the nine participants with active shoulder movement, the mean ROMs across the three phases were $99.9^{\circ}, 104.3^{\circ}$, and $126.3^{\circ}$, respectively. The nonparametric Friedman test for repeated measures ANOVA showed these differences to be statistically significant $(p=0.01)$, with the most significant difference between baseline 2 to postintervention $(p=0.02)$.

\section{Elbow Flexion Range of Motion}

Overall, no remarkable difference in ROM for elbow movement was recorded in participants from baseline to postintervention. While marked declines were recorded in participants $2\left(-4^{\circ}\right), 3\left(-13^{\circ}\right)$, and $6\left(-15^{\circ}\right)$, slight improvements were observed in participants $4,\left(5^{\circ}\right)$, $11\left(13^{\circ}\right)$, and $12\left(5^{\circ}\right)$. The mean ROM for the participants

Table 2.

Time spent with PreMotor Exercise Game intervention protocol (minutes).

\begin{tabular}{lrrrr}
\hline Participant & $\begin{array}{r}\text { Total } \\
\text { Time }\end{array}$ & $\begin{array}{r}\text { Puzzle } \\
\text { Game }\end{array}$ & $\begin{array}{r}\text { Time } \\
\text { Hand } \\
\text { Game }\end{array}$ & $\begin{array}{r}\text { Time } \\
\text { Arm }\end{array}$ \\
\hline 1 & 329.0 & 92.0 & 2.0 & 0.0 \\
2 & 221.0 & 98.0 & 34.0 & 0.0 \\
3 & $1,066.8$ & 180.0 & 368.0 & 149.0 \\
4 & 326.0 & 53.0 & 184.0 & 5.0 \\
5 & 743.5 & 30.0 & 30.0 & 350.0 \\
6 & 305.5 & 5.5 & 96.0 & 70.0 \\
7 & 527.0 & 168.0 & 165.0 & 109.0 \\
8 & 247.0 & 29.0 & 58.0 & 39.0 \\
9 & 934.0 & 186.0 & 430.0 & 71.0 \\
10 & $1,827.0$ & 417.0 & 544.0 & 265.0 \\
11 & $1,159.5$ & 198.5 & 341.0 & 211.0 \\
12 & $1,557.0$ & 421.0 & 479.0 & 297.0 \\
\hline \hline
\end{tabular}




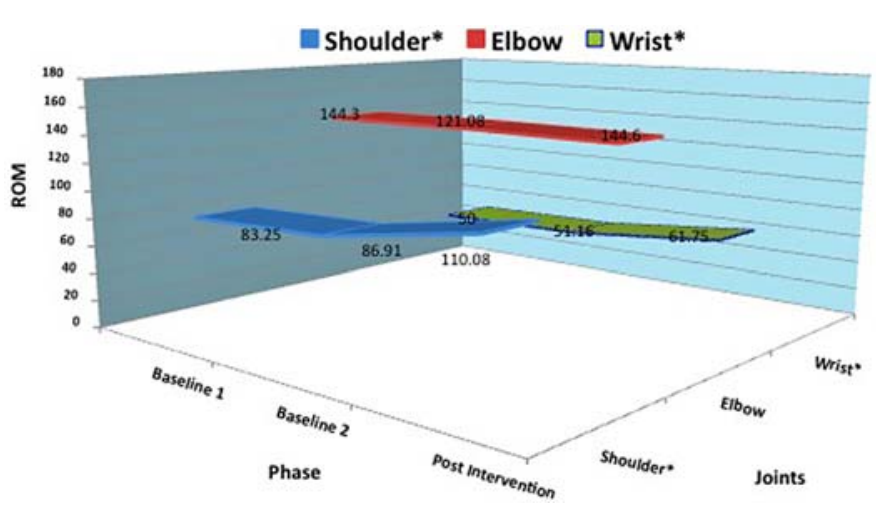

Figure 2.

Changes in range of motion (ROM). ${ }^{*} p<0.05$ (Friedman nonparametric repeated measures).

indicated a slight increase from the two baseline phases to postintervention correspondingly from $120.2^{\circ}, 121.0^{\circ}$, and $131.5^{\circ}$. These differences were not statistically significant for the Friedman test for repeated measures ANOVA and the nonparametric $t$-tests.

\section{Wrist Flexion Range of Motion}

The wrist ROM for the 11 participants who were assessed showed no difference to marked improvement. Although six of them indicated no changes in ROM from baseline to postintervention, improvements were recorded in five participants: $3,6,7,10$, and 11 . The difference for the sample was highest in the postintervention phase, with averages in $\mathrm{ROM}$ of $54^{\circ}, 55^{\circ}$, and $67^{\circ}$. The difference was statistically significant $(p=0.01)$ as analyzed by the Friedman test for repeated measures ANOVA. The difference was most significant from baseline 2 to postintervention, as indicated by a nonparametric $t$-test $(p=0.04)$.

Figure 3 illustrates the changes in strength of upperlimb muscles for participants from baseline to postintervention. Table 4 lists the change in strength for each participant.

\section{Shoulder (Flexion) Strength}

Following the PEGs intervention, no difference was seen with regards to the strength in shoulder flexion for the participants. Participants 3 and 6 showed marked improvements ranging from trace to fair strength, while participants 8 and 11 were tested from fair to normal strength. The difference for the sample was highest in the postintervention phase, with average shoulder flexion strength changing from 2.5 to 2.9. These differences were not statistically significant as analyzed by the Friedman test for repeated measures ANOVA.

No remarkable changes in elbow flexion strength were detected in the participants following the PEG intervention. However, similar to the findings with shoulder strength, participants 3,6 , and 8 showed improvements ranging from trace to poor, trace to fair, and good to normal, respectively. These improvements resulted in higher

Table 3.

Changes in range of motion for each participant.

\begin{tabular}{|c|c|c|c|c|c|c|}
\hline \multirow{2}{*}{ Participant } & \multicolumn{2}{|c|}{ Shoulder } & \multicolumn{2}{|c|}{ Elbow } & \multicolumn{2}{|c|}{ Wrist } \\
\hline & B1 to B2 & B2 to PI & B1 to B2 & B2 to PI & B1 to B2 & B2 to PI \\
\hline$\overline{1}$ & 0 & 0 & 0 & 0 & 0 & 0 \\
\hline 3 & 0 & +67 & 0 & -13 & 0 & +56 \\
\hline 4 & 0 & -2 & 0 & +5 & 0 & 0 \\
\hline 5 & - & - & 0 & +2 & 0 & 0 \\
\hline 7 & +22 & +16 & - & - & 0 & +15 \\
\hline 8 & +5 & +68 & 0 & 0 & 0 & 0 \\
\hline 9 & - & - & - & - & - & - \\
\hline 10 & +3 & +15 & 0 & 0 & +2 & +3 \\
\hline 11 & +4 & +53 & 0 & +13 & +2 & +8 \\
\hline
\end{tabular}


JRRD, Volume 51, Number 3, 2014

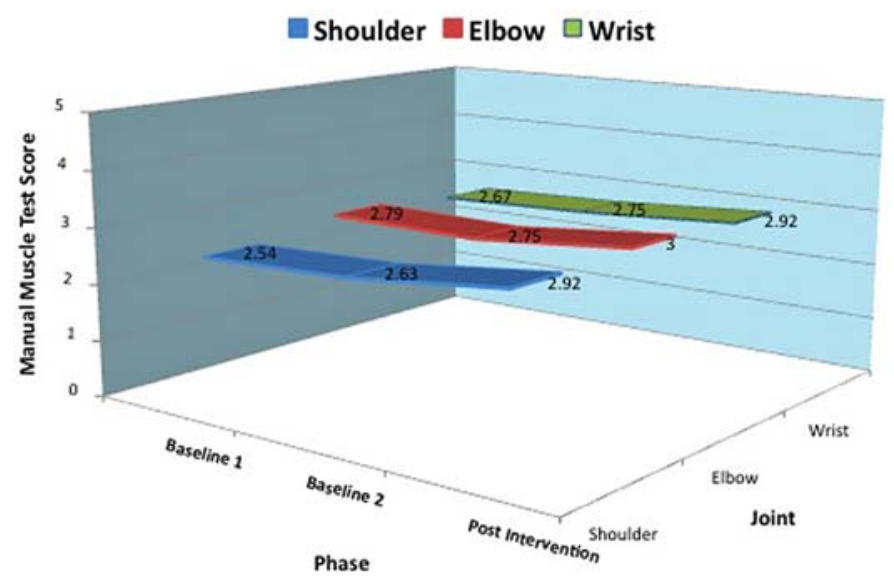

Figure 3.

Changes in muscle strength.

muscle strength average for the sample ranging from 2.8 to 3.0. Again, these differences were not statistically significant as analyzed by the Friedman test for repeated measures ANOVA.

The findings for wrist strength were similar to those of the shoulder and elbow in that the strength remained overall stable for the participants, with the exception of participants 3,6 , and 8 . The degree of improvement for these participants ranged similarly as before from trace to poor, trace to fair, and good to normal, respectively. As with findings for shoulder and elbow, participant 7 showed a decline in strength from poor to trace for wrist strength. The average wrist strength for the sample indicated an increase in the three phases from 2.6, 2.7, to 2.9, respectively, with the differences not being statistically significant.

The grip strength following the PEG intervention remained relatively stable for most participants. Participant 1 demonstrated the most improvement (12 lb), while participants 2, 11, and 15 were assessed for mild gains in grip strength. Overall, the average grip strength for the sample declined slightly from $42.08 \mathrm{lb}$ at second baseline to $41.83 \mathrm{lb}$ following intervention.

\section{Effect on Cognitive Skills}

All the participants achieved complete independence (score of 0) on the EFPT-initiation skill score following the PEG intervention, including participants 2, 3, 4, 5, 6, and 10 who demonstrated improvement from baseline to postintervention (Figure 4). As reflected in Figure 5, the mean differences for the 12 participants on the repeated measures Friedman analysis were significant $(p=0.01)$, with much of the significant change being accounted from baseline 2 to postintervention $(p=0.03)$.

In terms of execution skills, 10 out of 12 participants indicated improvement in scores on organization, with 8 participants gaining complete independence on EFPT tasks (Table 5). The differences were statistically significant $(p=0.01)$ on the Friedman analysis, and the change

Table 4.

Change in muscle strength for each participant.

\begin{tabular}{|c|c|c|c|c|c|c|}
\hline \multirow{2}{*}{ Participant } & \multicolumn{2}{|c|}{ Shoulder } & \multicolumn{2}{|c|}{ Elbow } & \multicolumn{2}{|c|}{ Wrist } \\
\hline & B1 to B2 & B2 to PI & B1 to $B 2$ & B2 to PI & B1 to B2 & B2 to PI \\
\hline$\overline{1}$ & +1.0 & 0.0 & 0.0 & 0.0 & +1.0 & 0.0 \\
\hline 2 & 0.0 & 0.0 & 0.0 & 0.0 & 0.0 & 0.0 \\
\hline 3 & 0.0 & +2.0 & 0.0 & +1.0 & 0.0 & +1.0 \\
\hline 4 & 0.0 & -1.0 & 0.0 & 0.0 & 0.0 & -1.0 \\
\hline 5 & 0.0 & 0.0 & 0.0 & 0.0 & 0.0 & 0.0 \\
\hline 6 & 0.0 & +2.0 & 0.0 & +2.0 & 0.0 & +2.0 \\
\hline 7 & 0.0 & -2.0 & 0.0 & -1.0 & 0.0 & -1.0 \\
\hline 8 & 0.0 & +1.0 & -0.1 & +1.0 & 0.0 & +1.0 \\
\hline 9 & - & - & - & - & - & - \\
\hline 10 & 0.0 & 0 & 0.0 & 0.0 & 0.0 & 0.0 \\
\hline 11 & 0.0 & +1.5 & 0.0 & 0.0 & 0.0 & 0.0 \\
\hline 12 & 0.0 & 0.0 & 0.0 & 0.0 & 0.0 & 0.0 \\
\hline Mean Difference & +0.1 & +0.3 & -0.1 & +0.3 & +0.1 & +0.2 \\
\hline
\end{tabular}




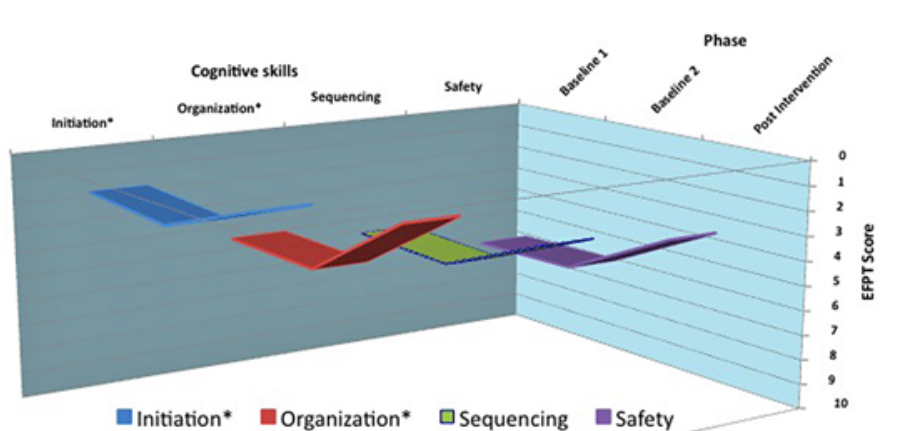

Figure 4.

Changes in Executive Function Performance Test (EFPT) skill scores. ${ }^{*} p<0.05$ (Friedman nonparametric repeated measures).

from baseline 2 to postintervention was significant ( $p=$ 0.04 ) as analyzed by the nonparametric $t$-test. The sequencing skills of 9 out of the 12 participants improved following the PEGs intervention, with 4 participants achieving a complete independence score. However, the differences across baseline to postintervention were not statistically significant. The scores on judgment and safety improved for 8 out of the 12 participants, with 5 participants demonstrating complete independence following intervention. The differences from baseline to postintervention were not statistically significant. However, the change in safety score was significant from baseline 2 to postintervention $(p<0.001)$.

For the overall EFPT task completion, 10 participants performed at the level of complete independence following the PEGs intervention, with 5 participants demonstrating improvement from the baseline phases. The mean differences from baseline to intervention were statistically significant $(p=0.02)$.

The EFPT task scores are presented in Figure 5. For cooking, all but one participant (11) demonstrated improvement in their scores, with six performing at independent level following the PEGs intervention (Table 6). The mean differences in improvement for this EFPT task were statistically significant $(p=0.02)$. For bill pay, 8 out of the 12 participants demonstrated improvement, which was noticeably evident in participants 1, 3, and 4. Although the mean differences reflect these improvements, they were not statistically significant $(p=0.07)$. The EFPT task for telephone use mostly remained unchanged for all participants, while improvements were substantial for the task of medication management, especially for 8 out of the 12 participants.

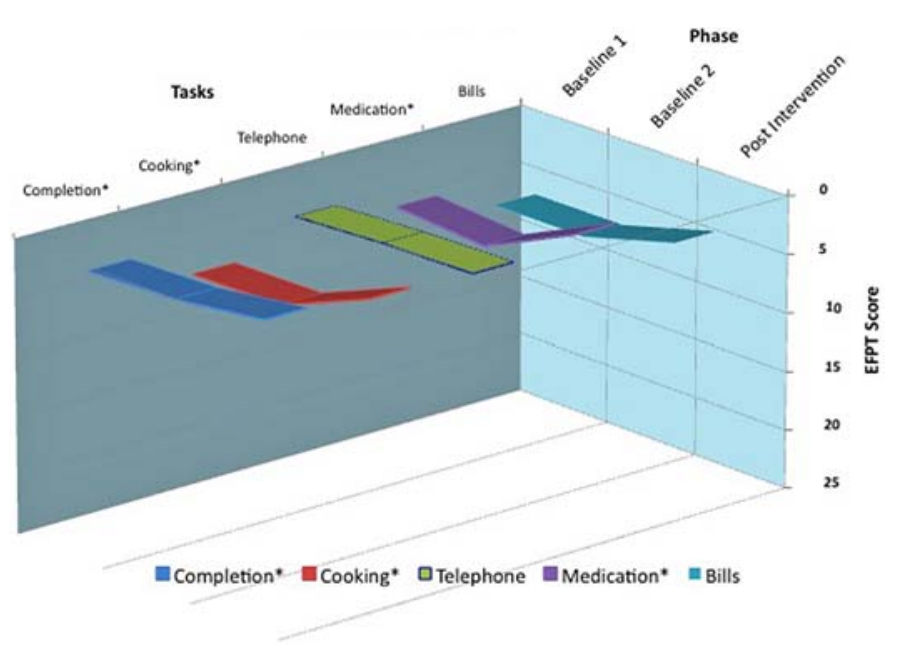

Figure 5.

Changes in Executive Function Performance Test (EFPT) task scores. ${ }^{*} p<0.05$ (Friedman nonparametric repeated measures).

The mean differences for scores on medication management from baseline to postintervention were statistically significant $(p=0.001)$, and the change was noted to be statistically significant from baseline 2 to postintervention $(p=0.01)$.

The task performance score for EFPT is presented in Figure 6. As seen in Table 7, nine participants demonstrated improvement in overall task performance, with the improvement noticeably evident in seven participants (1, $2,3,4,6,7$, and 8$)$. As a result, the mean difference on this global EFPT score was statistically significant $(p=0.02)$, with much of the gains being statistically significant between baseline 2 and postintervention $(p=0.03)$.

\section{DISCUSSION}

As seen in previous research, the participants in this study showed improvement in both ROM and strength for shoulder, elbow, and wrist flexion and grasp and pinch on the involved upper limb [25,27-29]. Although the gains were not statistically significant, the clinical/functional significance for improved upper-limb mobility should not be discounted. This was found in the activity demands of the EFPT, where participants were more able to open medicine bottles, reach and manipulate tools for cooking, and use a calculator for paying bills. The improvement in affected side grasp by most participants was intriguing because the demands of the intervention required significant contralateral manipulation of the computer mouse. The 
JRRD, Volume 51, Number 3, 2014

Table 5.

Changes in Executive Function Performance Test (EFPT)-Skill scores.

\begin{tabular}{|c|c|c|c|c|c|c|c|c|}
\hline \multirow{2}{*}{ Participant } & \multicolumn{2}{|c|}{ Initiation } & \multicolumn{2}{|c|}{ Organization } & \multicolumn{2}{|c|}{ Sequencing } & \multicolumn{2}{|c|}{ Safety } \\
\hline & B1 to B2 & B2 to PI & B1 to B2 & B2 to PI & B1 to B2 & B2 to PI & B1 to B2 & B2 to PI \\
\hline$\overline{1}$ & 0 & 0 & 0 & -7 & 0 & -2 & 0 & -8 \\
\hline 3 & 0 & -1 & 0 & -2 & 0 & 0 & 0 & -5 \\
\hline 4 & 0 & -3 & 0 & -9 & 0 & -11 & 0 & -11 \\
\hline 7 & 0 & 0 & 0 & -2 & 0 & -3 & 0 & -2 \\
\hline 8 & 0 & 0 & -1 & -1 & +3 & -4 & +4 & -5 \\
\hline 9 & 0 & 0 & +1 & +5 & +1 & +1 & 0 & +4 \\
\hline 10 & +1 & 1 & +2 & -3 & 0 & +1 & -3 & +3 \\
\hline 11 & 0 & 0 & 0 & -3 & -1 & +3 & -1 & +3 \\
\hline
\end{tabular}

Table 6.

Changes in Executive Function Performance Test (EFPT)-Task performance.

\begin{tabular}{|c|c|c|c|c|c|c|c|c|}
\hline \multirow{2}{*}{ Participant } & \multicolumn{2}{|c|}{ Cooking } & \multicolumn{2}{|c|}{ Telephone } & \multicolumn{2}{|c|}{ Medication } & \multicolumn{2}{|c|}{ Bills } \\
\hline & B1 to B2 & B2 to PI & B1 to B2 & B2 to PI & B1 to B2 & B2 to PI & B1 to B2 & B2 to PI \\
\hline$\overline{1}$ & 0 & -6 & 0 & -1 & 0 & -6 & 0 & -7 \\
\hline 3 & 0 & -5 & 0 & 0 & 0 & 0 & 0 & -3 \\
\hline 4 & 0 & -12 & 0 & -6 & 0 & -3 & 0 & -17 \\
\hline 5 & 0 & 0 & +1 & 0 & 0 & -2 & 0 & -1 \\
\hline 7 & 0 & +1 & 0 & 0 & 0 & -5 & 0 & -3 \\
\hline 8 & 0 & -1 & 0 & +1 & +8 & -8 & -3 & -2 \\
\hline 9 & 1 & -2 & 0 & +3 & 0 & 0 & 0 & +10 \\
\hline 10 & 0 & -4 & -5 & 0 & 0 & 0 & +3 & 0 \\
\hline 11 & 0 & +6 & 0 & +2 & -1 & -8 & 0 & +2 \\
\hline
\end{tabular}

motor demands of this activity (lateral key pinch and grasp) align with improvement on the involved side for pinch and grasp. Among the 12 participants, the total time spent on the PEG intervention ranged from 221 to $1,159 \mathrm{~min}$. We could not find any significant correlation between the changes in outcome measures and time spent on the inter- vention. Nonetheless, as in other studies, those participants who had higher levels of active movement during the pre-assessment phase seemed to benefit more from the treatment effects of the intervention.

It was interesting to note that the time spent during intervention did not correlate with motor gains. It appears 


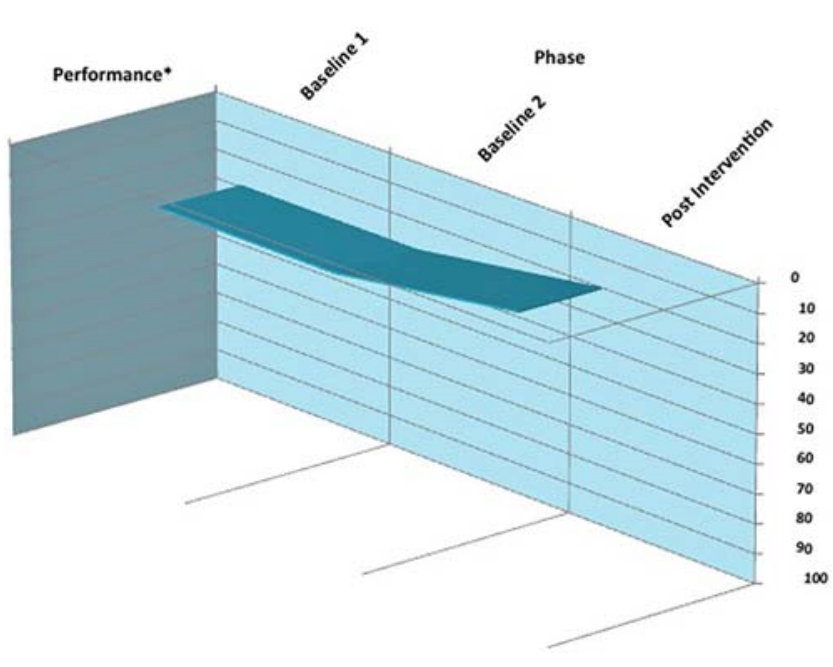

Figure 6.

Changes in Executive Function Performance Test (EFPT) overall task performance. ${ }^{*} p<0.05$ (Friedman nonparametric repeated measures).

that involvement in such a motor-based activity, even for short durations, is beneficial to those participants with ABI who had some level of active movement at pre-assessment.

Finally, from a motor perspective, it is important to note that the participants were, on average, $11.4 \mathrm{yr}$ postinjury and gains in joint movement of the shoulder, elbow, and wrist along with strength of the same joints and grasp and pinch were observed. Clearly, the study findings support the theoretical constructs of benefits associated with mirror therapy, mental imagery, and practice and computer-based motor intervention. These findings, with regards to this specific participant pool, add to a body of knowledge demonstrating that potential benefit and continued improvement years after injury are possible with involvement in focused motor intervention.

This study also addressed the concerns of Gutman et al. related to the cognitive demand of computer-based motor intervention [13]. Most participants demonstrated significant improvement in executive functioning skills. In consideration of the demands of the PEGs program, these findings have clinical implications. The PEGs program requires a significant initiation of task demand, as well as sequencing and problem-solving; therefore, it is critical for rehabilitation professionals to explain the broader benefit of this type of motor intervention to clients, especially those with ABI. The most important factor is that these interventions are transferable to daily activities such as preparing simple meals, taking medications, using the
Table 7.

Changes in Executive Function Performance Test (EFPT)-Overall task completion and performance.

\begin{tabular}{lccccc}
\hline \multirow{2}{*}{ Participant } & \multicolumn{2}{c}{ Completion } & & \multicolumn{2}{c}{ Performance } \\
\cline { 2 - 3 } \cline { 5 - 6 } & B1 to B2 & B2 to PI & & B1 to B2 & B2 to PI \\
\hline 1 & 0 & -3 & & 0 & -20 \\
2 & -1 & -2 & & +1 & -11 \\
3 & 0 & 0 & & 0 & -8 \\
4 & -1 & -4 & & 0 & -38 \\
5 & 0 & 0 & & +1 & -3 \\
6 & 0 & -4 & & 0 & -23 \\
7 & 0 & 0 & & -9 \\
8 & -1 & 0 & & +5 & -10 \\
9 & 0 & 0 & & -1 & +11 \\
10 & -2 & +1 & & -2 & -4 \\
11 & +1 & -1 & & -1 & +2 \\
12 & 0 & 0 & & -1 & 0 \\
Mean Difference & -0.3 & -1.1 & +0.2 & $-9.4^{*}$ \\
\hline Note: Negative score on EFPT is indicative of gain in cognitive skills. \\
$p_{p}<0.05$ (nonparametric Wilcoxon signed rank $t$-test). \\
B1 = baseline 1, B2 = baseline 2, PI = postintervention. \\
\hline \hline
\end{tabular}

phone, and paying bills. These are critical activities to most individuals, especially community-dwelling adults with ABI.

\section{LIMITATIONS}

In reviewing the results of this study, it is important to take into account the relatively small sample size and the need to replicate this intervention with a larger sample. The study participants averaged 11.4 chronic postinjury years, negating the possibility that neuroplasticity gains were due to other neuromotor interventions commonly seen in the in acute stages of recovery. Nonetheless, prior to drawing any clinical conclusions, a RCT involving individuals in acute to subacute stages of neuromotor recovery is warranted to verify how the study's findings generalize to that population.

Twelve adult volunteer participants were transported from Massachusetts, Maine, and within New Hampshire, three times each week. Attendance and time for actual intervention (simulation play) were determined by practical and personal factors experienced by the participants. Practical issues included cost to attend three times per week, dependency on and arrangements for transportation; reliance on availability of caregivers; and managing weather-related conditions, including New England road travel, sidewalk footing, and wheelchair malfunctions. 
These conditions influenced intervention time and should be considered in future studies.

\section{CONCLUSIONS}

The focus of this study was to determine whether individuals with chronic $\mathrm{ABI}$ could benefit from participating in a computer-based intervention focused on upper-limb and executive functioning. Although these findings are encouraging, more research is needed prior to integration of this type of intervention into the therapeutic process by rehabilitation professionals. However, computer-based, simulationdriven intervention could decrease demands on therapists and provide motivation for clients (related to the interest observed in most individuals for computer-based technology). Also, in an environment in which personal reimbursement for services is limited, insurance coverage is often inadequate, and treatment periods are short, this type of intervention, as evidenced in this study, can extend rehabilitation for individuals with motor involvement secondary to ABI. Further research involving this type of intervention and technology is indicated.

\section{ACKNOWLEDGMENTS}

\author{
Author Contributions: \\ Study concept and design: C. D. Simmons. \\ Acquisition of data: C. D. Simmons. \\ Analysis and interpretation of data: S. Arthanat, V. Macri. \\ Statistical analysis: S. Arthanat. \\ Drafting of manuscript: C. D. Simmons. \\ Critical revision of manuscript for important intellectual content: \\ V. Macri.
}

Financial Disclosures: The authors have declared that no competing interests exist.

Funding/Support: This work was unfunded at the time of manuscript preparation.

Institutional Review: Institutional review for human subject protection was conducted and approval was obtained from the center and through the Research Integrity Services at the University of New Hampshire.

Participant Follow-Up: The participants will be made aware of this publication through communication by authors to the community sites where they are currently working.

\section{REFERENCES}

1. Ruff R. Two decades of advances in understanding of mild traumatic brain injury. J Head Trauma Rehabil. 2005;20(1):
5-18. [PMID:15668567]

http://dx.doi.org/10.1097/00001199-200501000-00003

2. Faul M, Xu L, Wald MM, Corondo VG. Traumatic brain injury in the United States: Emergency department visits, hospitalizations and deaths 2002-2006. Atlanta (GA): Centers for Disease Control and Prevention; 2010.

3. Roger VL, Go AS, Lloyd-Joanes DM, Benjamin EJ, Berry J, Borden WB, Bravata DM, Dai S, Ford ES, Fox CS, Fullerton HJ, Gillespie C, Hailpern SM, Heit JA, Howard VJ, Kissela BM, Kittner SJ, Lackland DT, Lichtman JH, Lisabeth LD, Makuc DM, Marcus GM, Marelli A, Matchar DB, Moy CS, Mozaffarian D, Mussolino ME, Nichol G, Paynter NP, Soliman EZ, Sorlie PD, Sotoodehnia N, Turan TN, Virani SS, Wong ND, Woo D, Turner MB; American Heart Association Statistics Committee and Stroke Statistics Subcommittee. Heart disease and stroke statistics-2012 update: A report from the American Heart Association. Circulation. 2012;125:2-20. [PMID:22179539] http://dx.doi.org/10.1161/CIR.0b013e31823ac046

4. Chen HF, Wu CY, Lin KC, Chen HC, Chen CP, Chen CK. Rasch validation of the streamlined Wolf Motor Function Test in people with chronic stroke and subacute stroke. Phys Ther. 2012;92(8):1017-26. [PMID:22556276] http://dx.doi.org/10.2522/ptj.20110175

5. Kwakkel G, Kollen BJ, van der Grond J, Prevo AJ. Probability of regaining dexterity in the flaccid upper limb: Impact of severity of paresis and time since onset in acute stroke. Stroke. 2003;34(9):2181-86. [PMID:12907818] http://dx.doi.org/10.1161/01.STR.0000087172.16305.CD

6. Gallese V, Goldman A. Mirror neurons and the simulation theory of mind-reading. Trends Cogn Sci. 1998;2(12): 493-501. [PMID:21227300]

7. Gallese V, Fadiga L, Fogassi L, Rizzolatti G. Action recognition in the premotor cortex. Brain. 1996;119(2):593-609. [PMID:8800951] http://dx.doi.org/10.1093/brain/119.2.593

8. Ferrari PF, Gallese V, Rizzolatti G, Fogassi L. Mirror neurons responding to the observation of ingestive and communicative mouth actions in the monkey ventral premotor cortex. Eur J Neurosci. 2003;17(8):1703-14.

[PMID:12752388]

http://dx.doi.org/10.1046/j.1460-9568.2003.02601.x

9. Raos V, Evangeliou MN, Savaki HE. Observation of action: Grasping with the mind's hand. Neuroimage. 2004; 23(1):193-201. [PMID:15325366] http://dx.doi.org/10.1016/j.neuroimage.2004.04.024

10. Rizzolatti G, Fadiga L, Gallese V, Fogassi L. Premotor cortex and the recognition of motor actions. Brain Res Cogn Brain Res. 1996;3(2):131-41. [PMID:8713554] http://dx.doi.org/10.1016/0926-6410(95)00038-0 
11. Rizzolatti G, Craighero L. The mirror-neuron system. Annu Rev Neurosci. 2004;27:169-92. [PMID:15217330] http://dx.doi.org/10.1146/annurev.neuro.27.070203.144230

12. Rizzolatti G, Fogassi L, Gallese V. Mirrors of the mind. Sci Am. 2006;295(5):54-61. [PMID:17076084] http://dx.doi.org/10.1038/scientificamerican1106-54

13. Gutman SA, Raphael EI, Ceder LM, Khan A, Timp KM, Salvant S. The effect of a motor-based, social skills intervention for adolescents with high-functioning autism: Two single-subject design cases. Occup Ther Int. 2010;17(4): 188-97. [PMID:20672254] http://dx.doi.org/10.1002/oti.300

14. Pomeroy VM, Clark CA, Miller JS, Baron JC, Markus HS, Tallis RC. The potential for utilizing the "mirror neurone system" to enhance recovery of the severely affected upper limb early after stroke: A review and hypothesis. Neurorehabil Neural Repair. 2005;19(1):4-13. [PMID:15673838] http://dx.doi.org/10.1177/1545968304274351

15. Blakemore SJ, Decety J. From the perception of action to the understanding of intention. Nat Rev Neurosci. 2001; 2(8):561-67. [PMID:11483999]

16. Ramachandran VS. The tell-tale brain: A neuroscientist's quest for what makes us human. New York (NY): W.W. Norton; 2011.

17. Michielsen ME, Smits M, Ribbers GM, Stam HJ, van der Geest JN, Bussmann JB, Selles RW. The neuronal correlates of mirror therapy: An fMRI study on mirror induced visual illusions in patients with stroke. J Neurol Neurosurg Psychiatry. 2011;82(4):393-98. [PMID:20861065] http://dx.doi.org/10.1136/jnnp.2009.194134

18. Thieme H, Mehrholz J, Pohl M, Behrens J, Dohle C. Mirror therapy for improving motor function after stroke. Cochrane Database Syst Rev. 2012;3:CD008449.

19. Altschuler EL, Wisdom SB, Stone L, Foster C, Galasko D, Llewellyn DM, Ramachandran VS. Rehabilitation of hemiparesis after stroke with a mirror. Lancet. 1999;353(9169): 2035-36. [PMID:10376620] http://dx.doi.org/10.1016/S0140-6736(99)00920-4

20. Stevens JA, Stoykov ME. Using motor imagery in the rehabilitation of hemiparesis. Arch Phys Med Rehabil. 2003; 84(7):1090-92. [PMID:12881842] http://dx.doi.org/10.1016/S0003-9993(03)00042-X

21. Lee MM, Cho HY, Song CH. The mirror therapy program enhances upper-limb motor recovery and motor function in acute stroke patients. Am J Phys Med Rehabil. 2012; 91(8):689-96, quiz 697-700. [PMID:22469877] http://dx.doi.org/10.1097/PHM.0b013e31824fa86d

22. Holden MK, Dyar T. Virtual environment training: A new tool for neurorehabilitation. J Neuro Phys Ther. 2002; 26(2):62-71.

23. Wille D, Eng K, Holper L, Chevrier E, Hauser Y, Kiper D, Pyk P, Schlegel S, Meyer-Heim A. Virtual reality-based paediatric interactive therapy system (PITS) for improvement of arm and hand function in children with motor impairment — a pilot study. Dev Neurorehabil. 2009;12(1): 44-52. [PMID:19283533] http://dx.doi.org/10.1080/17518420902773117

24. Butler AJ, Page SJ. Mental practice with motor imagery: Evidence for motor recovery and cortical reorganization after stroke. Arch Phys Med Rehabil. 2006;87(12 Suppl 2):S2-11. [PMID:17140874] http://dx.doi.org/10.1016/j.apmr.2006.08.326

25. Lacourse MG, Turner JA, Randolph-Orr E, Schandler SL, Cohen MJ. Cerebral and cerebellar sensorimotor plasticity following motor imagery-based mental practice of a sequential movement. J Rehabil Res Dev. 2004;41(4):505-24.

[PMID:15558380] http://dx.doi.org/10.1682/JRRD.2004.04.0505

26. Vromen A, Verbunt JA, Rasquin S, Wade DT. Motor imagery in patients with a right hemisphere stroke and unilateral neglect. Brain Inj. 2011;25(4):387-93. [PMID:21355672] http://dx.doi.org/10.3109/02699052.2011.558041

27. Calayan LS, Dizon JM. A systematic review on the effectiveness of mental practice with motor imagery in the neurologic rehabilitation of stroke patients. Internet $\mathrm{J}$ Allied Health Sci Pract. 2009;7:1-11.

28. Braun SM, Beurskens AJ, Borm PJ, Schack T, Wade DT. The effects of mental practice in stroke rehabilitation: A systematic review. Arch Phys Med Rehabil. 2006;87(6): 842-52. [PMID:16731221] http://dx.doi.org/10.1016/j.apmr.2006.02.034

29. Hong IK, Choi JB, Lee JH. Cortical changes after mental imagery training combined with electromyography-triggered electrical stimulation in patients with chronic stroke. Stroke. 2012;43(9):2506-9. [PMID:22798329] http://dx.doi.org/10.1161/STROKEAHA.112.663641

30. Malouin F, Richards CL. Mental practice for relearning locomotor skills. Phys Ther. 2010;90(2):240-51. [PMID:20022993] http://dx.doi.org/10.2522/ptj.20090029

31. Sharma N, Pomeroy VM, Baron JC. Motor imagery: A backdoor to the motor system after stroke? Stroke. 2006; 37(7):1941-52. [PMID:16741183] http://dx.doi.org/10.1161/01.STR.0000226902.43357.fc

32. Howell DC. Fundamentals statistics for the behavioral sciences. 6th ed. Belmont (CA): Thomson Wadsworth; 2008.

33. Portney LG, Watkins MP. Foundation of clinical research: Applications to practice. 2nd ed. Englewood Cliffs (NJ): Prentice-Hall; 2000.

34. Baum CM, Connor LT, Morrison T, Hahn M, Dromerick AW, Edwards DF. Reliability, validity, and clinical utility of the Executive Function Performance Test: A measure of executive function in a sample of people with stroke. Am J 
JRRD, Volume 51, Number 3, 2014

Occup Ther. 2008;62(4):446-55. [PMID:18712007]

http://dx.doi.org/10.5014/ajot.62.4.446

Submitted for publication May 3, 2013. Accepted in revised form November 26, 2013.

This article and any supplementary material should be cited as follows:

Simmons CD, Arthanat S, Macri VJ. Pilot study: Computer- based virtual anatomical interactivity for rehabilitation of individuals with chronic acquired brain injury. J Rehabil Res Dev. 2014;51(3):377-90.

http://dx.doi.org/10.1682/JRRD.2013.05.0103

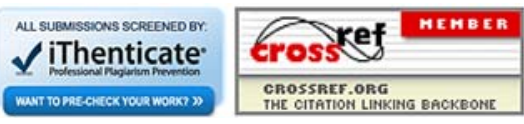

